\title{
Lack of an association between SCFD1 rs10139154 polymorphism and amyotrophic lateral sclerosis
}

\author{
VASILEIOS SIOKAS $^{1}$, ATHINA-MARIA ALOIZOU ${ }^{1}$, IOANNIS LIAMPAS ${ }^{1}$, CHRISTOS BAKIRTZIS $^{2}$, \\ GRIGORIOS NASIOS $^{3}$, KONSTANTINOS PATERAKIS ${ }^{4}$, MARKOS SGANTZOS ${ }^{1}$, \\ DIMITRIOS P. BOGDANOS ${ }^{5}$, DEMETRIOS A. SPANDIDOS ${ }^{6}$, ARISTIDIS TSATSAKIS ${ }^{7}$, \\ PANAYIOTIS D. MITSIAS ${ }^{8-10}$ and EFTHIMIOS DARDIOTIS ${ }^{1}$
}

\begin{abstract}
${ }^{1}$ Department of Neurology, Laboratory of Neurogenetics, University Hospital of Larissa, Faculty of Medicine, School of Health Sciences, University of Thessaly, 41100 Larissa; ${ }^{2} \mathrm{~B}^{\prime}$ Department of Neurology, AHEPA University Hospital, Aristotle University of Thessaloniki, 54636 Thessaloniki; ${ }^{3}$ Department of Speech and Language Therapy, University of Ioannina, 45500 Ioannina; ${ }^{4}$ Department of Neurosurgery, University Hospital of Larissa, Medical School, University of Thessaly; ${ }^{5}$ Department of Rheumatology and Clinical Immunology, University General Hospital of Larissa, Faculty of Medicine, School of Health Sciences, University of Thessaly, 41100 Larissa; ${ }^{6}$ Laboratory of Clinical Virology, ${ }^{7}$ Laboratory of Toxicology, and ${ }^{8}$ Department of Neurology, School of Medicine, University of Crete, 71003 Heraklion, Greece;

${ }^{9}$ Department of Neurology, Henry Ford Hospital; ${ }^{10}$ School of Medicine, Wayne State University, Detroit, MI 48202, USA
\end{abstract}

Received January 12, 2022; Accepted February 15, 2022

DOI: $10.3892 / \mathrm{mmr} .2022 .12662$

\begin{abstract}
Amyotrophic lateral sclerosis (ALS) is a progressive neurodegenerative disease. Through a genome-wide association study (GWAS), the Sec1 family domain-containing protein 1 (SCFD1) rs10139154 variant at 14q12 has emerged as a risk factor gene for ALS. Moreover, it has been reported to influence the age at onset (AAO) of patients with ALS. The aim of the present study was to assess the association of the SCFD1 rs10139154 polymorphism with the risk of developing ALS. For this purpose, 155 patients with sporadic ALS and 155 healthy controls were genotyped for the SCFD1 rs10139154. The effect of the SCFD1 rs10139154 polymorphism was then examined on the following parameters: i) The risk of developing ALS; ii) the AAO of ALS; iii) the site of ALS onset (patients with bulbar onset ALS vs. healthy controls; and patients with limb onset ALS vs. healthy controls); and iv) the AAO of ALS onset with subgroup analyses based on the site of onset (bulbar and limb, crude and adjusted for sex). The analysis of all the outcomes was performed assuming five genetic models. Crude and adjusted analyses were applied. The threshold for statistical significance was set at 0.05 . The results revealed no association between SCFD1 rs10139154 and any of
\end{abstract}

Correspondence to: Dr Efthimios Dardiotis, Department of Neurology, Laboratory of Neurogenetics, University Hospital of Larissa, Faculty of Medicine, School of Health Sciences, University of Thessaly, Biopolis, Mezourlo Hill, 41100 Larissa, Greece E-mail: edar@med.uth.gr; ebsdar@gmail.com

Key words: amyotrophic lateral sclerosis, polymorphism, Sec1 family domain-containing protein 1, rs10139154, genetics the examined phenotypes in any of the models examined. On the whole, based on the findings of the present study, SCFD1 rs10139154 does not appear to play a determining role in the risk of developing ALS.

\section{Introduction}

Amyotrophic lateral sclerosis (ALS) is a neurodegenerative disease of a yet undetermined etiology, and it is estimated to be the most common type of motor neuron disease during adulthood (1). A great variation has been observed in terms of the frequency of ALS between different countries, with males exhibiting a slightly elevated risk of developing ALS compared with females, particularly as regards the form of ALS with limb onset (2-4). On the other hand, there is a slight female predominance in bulbar onset ALS (2-4).

ALS can be phenotypically manifested with symptomatology from the upper (e.g., spasticity, weakness, hyperreflexia and pseudobulbar palsy) and lower (e.g. atrophy of muscles, fasciculations, hyporeflexia and muscle cramps) motor neurons (5). The consequent progressive and irreversible muscular weakness and atrophy, leads to the paralysis of respiratory muscles and respiratory failure (6). Apart from motor deficits, non-motor symptoms [e.g., frontotemporal dementia (FTD)] can also develop with ALS (7). The association between ALS and FTD is evident through the crosstalk that exists between them clinically, genetically and neuropathologically (8).

From a neuropathological aspect, ALS presents as a degeneration of motor neurons, while intraneuronal inclusions in glial cells and neurons can also be observed (9). While the etiology and the exact mechanisms that possible lead to the development of ALS remain to be determined, a notable amount of research suggests that complex mechanisms, 
that interact with each other, are possibly implicated in the development of ALS $(9,10)$. Mitochondrial dysfunction, inflammatory abnormalities, pathological protein aggregation, defective microtubule function, inadequate RNA activity, oxidative stress and glutamate excitotoxicity, to mention a few, are among the pathological processes that have been found to confer susceptibility to ALS $(11,12)$.

The molecular pathways through which the aforementioned mechanisms can lead to ALS are far from being completely elucidated (13). However, there are a number of indications that genetic, environmental and epigenetic factors all contribute to ALS susceptibility (14). Among the exogenous factors, head trauma, smoking, viral infections, exposure to pesticides and heavy metals, antioxidants, physical exercise, exposure to electromagnetic fields and body mass index have been previously reported as possible triggers for the development of ALS (15,16).

The contribution of genetic factors to ALS is supported from robust data (17). Firstly, there is familial ALS (fALS; accounting for almost 5-10\% of all ALS cases), where mutations $>30$ genes are considered as ALS causative ones $(10,18)$. Namely, mutations in the chromosome 9 open reading frame 72 (C9orf72), superoxide dismutase type 1 (SOD1), TAR DNA-binding protein 43 (TDP-43) and fused in sarcoma/translocated in liposarcoma (FUS/TLS) genes are estimated to be the commonest ones $(10,18)$. Mutations in these genes can lead to ALS; however, there are some genotype-phenotype associations, where patients with ALS carrying specific mutations may exhibit specific sub-phenotypes (19). For example, C9orf72 can lead to ALS, behavioral variant FTD, or ALS-FTD, while mutations in the TDP43 gene can lead to ALS with either bulbar or limb onset (19). Moreover, the remaining $85-90 \%$ of all ALS cases are considered as sporadic ALS (sALS) (5), where a large amount of genetic loci have been reported to modify the risk of developing ALS (20). At this point, it is worth mentioning that the conferred risk from rare genetic variants suggests an oligogenic disease pattern concerning the ALS architecture $(21,22)$.

The Sec1 family domain containing 1 (SCFD1) protein, belongs to the Senc1/Munk 18 (SM) family of proteins (23). These proteins are vesicle-trafficking proteins, working closely with a particular type of SNARE proteins, the syntaxins $(24,25)$. SCFD1 is implicated in a number of functions, such as cellular membrane fusion, oxidative-stress, intra-Golgi-retrograde transport and apoptosis (23-26).

In 2016, a genome-wide association study (GWAS) identified the SCFD1 rs10139154 variant at 14q12 as a genetic locus associated with ALS in the discovery phase; however, statistical significance was not found in the replication phase (27). Moreover, recently, Chen et al (28) examined the effect of the SCFD1 rs10139154 on a large Chinese population with ALS. Based on the results of that study, the SCFD1 rs10139154 polymorphism was shown to be associated with the age at onset (AAO) of patients with ALS.

Taking into consideration that SCFD1 is involved in mechanisms (23-26) that are also possibly implicated in ALS pathogenesis (e.g., oxidative stress and apoptosis) $(11,12)$, and that the studies examining the role of the SCFD1 rs10139154 variant regarding ALS have yielded inconsistent results $(27,28)$, the present case-control study was conducted with the aim of determining any possible association between the SCFD1 rs10139154 variant and ALS. A Southeastern European Caucasian (SEC) cohort (Greek) was analyzed, primary aiming at detecting any association between SCFD1 rs10139154 and ALS. The present study also wished to examine the effect of this polymorphism on several other ALS endophenotypes.

\section{Patients and methods}

Study participants. In the current protocol, a total of 310 participants were drafted. In greater detail, 155 patients with sporadic ALS, as well as 155 healthy controls were included. The participants were recruited between March, 2017 and September, 2017 from the University Hospital of Larissa (Department of Neurology), in Larissa, Greece. Consultant neurologists made the diagnoses of ALS, following the El Escorial criteria (29). The inclusion criteria for the participants with ALS were as follows: i) An age >18 years; ii) Greek ethnicity; and iii) a diagnosis of ALS based on the El Escorial criteria. The respective inclusion criteria for healthy controls were the following: i) An age >18 years; ii) of Greek ethnic origin; and iii) no referred family or personal history of ALS or other neurological disorders. The characteristics of the ALS cohort have been previously described (30-33). The research study protocol of the present study was approved by the Local Ethics Committee (University Hospital of Larissa: 59295/23-01-2017) and it was deemed in accordance to the Declaration of Helsinki. The experimental protocol was explained and all the participants provided their written informed consent in order to be included in the study, with the freedom of withdrawing from the study at any given time.

Molecular genetics. The salting out method was used to isolate genomic DNA from leucocytes (blood was collected with peripheral venipuncture from each participant), a method which has also been previously described $(34,35)$. All the samples derived from the healthy volunteers and the patients with ALS were genotyped for the SCFD1 rs10139154 variant. The method which was applied for genotyping was the TaqMan allele specific discrimination assay on an ABI PRISM 7900 Sequence Detection System. The results were then analyzed using SDS software (SDS 2.4). (Applied Biosystems; Thermo Fisher Scientific, Inc.). In brief, this method consisted of an initial enzyme activation occurring as the first step of PCR at a $50^{\circ} \mathrm{C}$ incubation for $2 \mathrm{~min}$, followed by enzyme activation at $95^{\circ} \mathrm{C}$ for $10 \mathrm{~min}$ (one cycle), denaturation at $95^{\circ} \mathrm{C}$ for $15 \mathrm{sec}$ and annealing/extension at $60^{\circ} \mathrm{C}$ for $1 \mathrm{~min}(40$ cycles for the last two points). The personnel who performed the genotyping was blinded to status of the samples.

Quality assessment. In order for the genotyping reproducibility be evaluated, a random $10 \%$ of the sample was re-genotyped, with $100 \%$ concordance. Moreover, the threshold for the genotype call rate (percentage of successfully genotyped samples) was set as $\geq 95 \%$. Additionally, the deviation or not from the Hardy-Weinberg equilibrium (HWE) was calculated with the Chi-squared test (36), in both the patients with ALS and the healthy controls. 
Statistical analysis. The CaTS Power Calculator for Genetic Studies (Center for Statistical Genetics, University of Michigan, Ann Arbor, MI, USA) was used for the statistical power of the current sample to be measured. With odds ratios (ORs) along with the respective $95 \%$ confidence intervals (CIs), the study sample was examined for associations between SCFD1 rs10139154 and ALS using SNPStats software (https://www. snpstats.net/) (36). Towards this, five genetic models were assumed: i) The co-dominant model, where a P-value with 2 degrees of freedom and 2 ORs were calculated [OR1: $(\mathrm{mt} / \mathrm{wt}$ vs. wt/wt) and OR2: (mt/mt vs. wt/wt)]; ii) the dominant model $(\mathrm{mt} / \mathrm{mt}+\mathrm{mt} / \mathrm{wt}$ vs. wt/wt); iii) the recessive model $(\mathrm{mt} / \mathrm{mt}$ vs. $\mathrm{mt} / \mathrm{wt}+\mathrm{wt} / \mathrm{wt}) ; \mathrm{iv})$ the overdominant model (mt/wt vs. $\mathrm{mt} / \mathrm{mt}+\mathrm{wt} / \mathrm{wt}$ ) and $\mathrm{v}$ ) the log-additive model, where $\mathrm{mt} / \mathrm{mt}$ carriers have a double risk of disease compared with $\mathrm{mt} / \mathrm{wt}$, with wt/wt as a reference. In the analyses, the ' $C$ ' was defined as the wild-type allele (wt) and the ' $\mathrm{T}$ ' as the mutant allele (mt), for the SCFD1 rs10139154 variant. A P-value <0.05 was considered to indicate a statistically significant difference. Both univariate (unadjusted) and multivariate (adjusted for age and sex) analyses were performed.

The primary outcome of the protocol of the present study was to examine the possible association between ALS and SCFD1 rs10139154 (ALS vs. healthy controls). Unadjusted and adjusted for age and sex analyses were performed. In addition, the effects of SCFD1 rs10139154 on the following parameters were examined: i) The AAO of ALS (unadjusted and adjusted for sex); ii) the site of ALS onset (patients with bulbar onset ALS vs. healthy controls; and patients with limb onset ALS vs. healthy controls; unadjusted and adjusted for age and sex); and iii) the AAO of ALS with subgroup analyses on the basis of the site of onset (bulbar and limb, crude and adjusted for sex). The analysis of all the outcomes was performed on the basis of the aforementioned genetic models.

\section{Results}

A total of 310 individuals were recruited in the present study; 155 patients with definite ALS [77 (49.7\%) female, age (mean \pm standard deviation), 63.74 \pm 11.30 years], and 155 healthy volunteers (control group) matched for age and sex. Approximately 7 out of $10(67.1 \%)$ patients with ALS confirmed a history of alcohol consumption, while $68.4 \%$ had a history of smoking. The most common site of ALS onset was the lower limbs (34.8\%) and the bulbar type (32.3\%). The characteristics of the patients with ALS have been formerly reported (30-32).

The genotype call rate was $98.06 \%$ (304/310). Furthermore, no deviation from the HWE was observed in both patients with ALS and the healthy controls $(\mathrm{P}=0.37$ and $\mathrm{P}=0.23$, respectively) (Table I). The analysis for the power of the sample size of the study was 80.4 to detect a significant association $(\mathrm{P}<0.05)$ between ALS and SCFD1 rs10139154, with the frequency of the minor T allele set at $37 \%$, an ALS prevalence of 5/100,000, and an approximate relative risk of 1.59 for the multiplicative mode of inheritance.

The frequency of the minor allele (T) was 35 and $38 \%$ for the patients with ALS and healthy controls respectively. The genotypic frequencies $\mathrm{C} / \mathrm{C}, \mathrm{C} / \mathrm{T}$ and $\mathrm{T} / \mathrm{T}$ for the patients with ALS, were 44, 42 and 14\%, respectively, while in the group of
Table I. Results from the Chi-squared exact test for HWE for the SCFD1 rs 10139154 in healthy controls and in ALS cases.

\begin{tabular}{lcr}
\hline \multirow{2}{*}{ SNP } & \multicolumn{2}{c}{ P-value } \\
\cline { 2 - 3 } rs10139154 & 0.23 & ALS \\
\hline $\begin{array}{l}\text { HWE, Hardy-Weinberg equilibrium; SNP, single nucleotide poly- } \\
\text { morphism; ALS, amyotrophic lateral sclerosis; SCFD1, Sec1 family } \\
\text { domain containing 1. }\end{array}$ & 0.37 \\
\hline
\end{tabular}

healthy volunteers, the corresponding frequencies were 41,42 and $17 \%$, respectively. The allelic and genotypic frequencies for SCFD1 rs10139154, for both patients with ALS and the healthy controls are presented in Table II. The allele and genotype numbers for SCFD1 rs10139154 in the healthy controls and ALS cases are graphically presented in Figs. 1 and 2, respectively.

Based on the univariate (unadjusted) and multivariate (adjusted for age and sex) analyses, no significant association was found between the SCFD1 rs10139154 polymorphism and ALS in any genetic model examined (Table III). Subgroup analysis (unadjusted and adjusted for age and sex) according to the site of ALS onset (limb, bulbar) also did not reveal any significant association (Table IV).

Furthermore, the analyses based on the AAO of ALS (unadjusted and adjusted for sex), failed to produce any statistically significant differences. The analyses for the AAO of ALS with regards to the site of onset (limb, bulbar), also revealed non-statistically significant results. The respective results (ORs, CIs, P-values, mean and standard error of the age for each genotype) are presented in Table V, for the ALS group overall. The results from the subgroup analysis regarding the AAO, based on the site of the ALS onset are presented in Table VI for the bulbar onset and in Table VII for the limb onset.

\section{Discussion}

The present case-control study genotyped an ALS cohort with SEC ancestry aiming to examine the role of the SCFD1 rs10139154 polymorphism in ALS. Based on the results obtained, the SCFD1 rs10139154 polymorphism was not associated with ALS. More precisely, the SCFD1 rs10139154 polymorphism neither conferred any susceptibility to ALS nor influenced the AAO of ALS or its initial manifestation. Therefore, based on this analysis, it appears rather unlikely that the SCFD1 rs10139154 polymorphism is among the risk factors for the development of ALS.

As aforementioned, the SCFD1 is a vesicle-trafficking protein, functioning alongside with syntaxins $(24,25)$. The possible mechanisms via which SCFD1 may be related to ALS pathophysiology are cellular membrane fusion, oxidative-stress, intra-Golgi-retrograde transport and apoptosis (23-26). The SCFD1 rs10139154 polymorphism is an intronic variant located at chromosome 14: 30678292 (https://www.ensembl. 
Table II. Allelic and genotype frequencies for SCFD1 rs10139154 in the healthy controls, ALS cases and the whole sample size.

\begin{tabular}{|c|c|c|c|c|}
\hline rs10139154 SNP & $\begin{array}{l}\text { Genotypes/ } \\
\text { alleles }\end{array}$ & $\begin{array}{l}\text { Healthy controls } \\
(\mathrm{n}=155), \mathrm{n}(\%)\end{array}$ & $\begin{array}{c}\text { ALS } \\
(\mathrm{n}=155), \mathrm{n}(\%)\end{array}$ & $\begin{array}{l}\text { Whole sample } \\
(\mathrm{n}=310), \mathrm{n}(\%)\end{array}$ \\
\hline \multirow[t]{4}{*}{ Genotype } & $\mathrm{C} / \mathrm{C}$ & $62(41)$ & $67(44)$ & $129(42)$ \\
\hline & $\mathrm{C} / \mathrm{T}$ & $64(42)$ & $64(42)$ & $128(42)$ \\
\hline & $\mathrm{T} / \mathrm{T}$ & $26(17)$ & $21(14)$ & $47(15)$ \\
\hline & Missing & 3 & 3 & 6 \\
\hline \multirow[t]{2}{*}{ Allele } & $\mathrm{C}$ & $188(62)$ & $198(65)$ & $386(63)$ \\
\hline & $\mathrm{T}$ & $116(38)$ & $106(35)$ & $222(37)$ \\
\hline
\end{tabular}

SNP, single nucleotide polymorphism; ALS, amyotrophic lateral sclerosis; SCFD1, Sec1 family domain containing 1.

Table III. Single locus analysis (crude and adjusted for age and sex) for association between SCFD1 rs10139154 and ALS, in co-dominant, dominant, recessive, overdominant and log-additive mode.

\begin{tabular}{|c|c|c|c|c|c|}
\hline \multirow[b]{2}{*}{ Mode } & \multirow[b]{2}{*}{ Genotype } & \multicolumn{2}{|c|}{ Univariate } & \multicolumn{2}{|c|}{ Multivariate } \\
\hline & & OR $(95 \% \mathrm{CI})$ & P-value & OR $(95 \% \mathrm{CI})$ & P-value \\
\hline \multirow[t]{3}{*}{ Co-dominant } & $\mathrm{C} / \mathrm{C}$ & 1.00 & \multirow[t]{3}{*}{0.7} & 1.00 & \multirow[t]{3}{*}{0.44} \\
\hline & $\mathrm{C} / \mathrm{T}$ & $0.93(0.57-1.51)$ & & $0.77(0.38-1.54)$ & \\
\hline & $\mathrm{T} / \mathrm{T}$ & $0.75(0.38-1.46)$ & & $0.57(0.23-1.39)$ & \\
\hline \multirow[t]{2}{*}{ Dominant } & $\mathrm{C} / \mathrm{C}$ & 1.00 & \multirow[t]{2}{*}{0.56} & 1.00 & \multirow[t]{2}{*}{0.28} \\
\hline & $\mathrm{C} / \mathrm{T}-\mathrm{T} / \mathrm{T}$ & $0.87(0.55-1.38)$ & & $0.70(0.37-1.33)$ & \\
\hline \multirow[t]{2}{*}{ Recessive } & $\mathrm{C} / \mathrm{C}-\mathrm{C} / \mathrm{T}$ & 1.00 & \multirow[t]{2}{*}{0.43} & 1.00 & \multirow[t]{2}{*}{0.3} \\
\hline & $\mathrm{T} / \mathrm{T}$ & $0.78(0.42-1.45)$ & & $0.64(0.28-1.48)$ & \\
\hline \multirow[t]{2}{*}{ Overdominant } & $\mathrm{C} / \mathrm{C}-\mathrm{T} / \mathrm{T}$ & 1.00 & \multirow[t]{2}{*}{0.99} & 1.00 & \multirow[t]{2}{*}{0.78} \\
\hline & $\mathrm{C} / \mathrm{T}$ & $1.00(0.63-1.58)$ & & $0.91(0.48-1.73)$ & \\
\hline Log-additive & - & $0.88(0.64-1.21)$ & 0.42 & $0.76(0.49-1.16)$ & 0.2 \\
\hline
\end{tabular}

SCFD1, Sec1 family domain containing 1; ALS, amyotrophic lateral sclerosis; CI, confidence interval; OR, odds ratio.

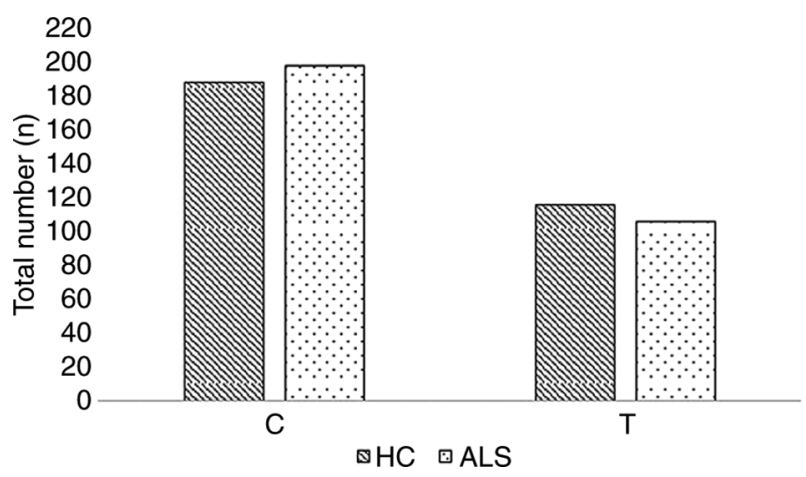

Figure 1. Allele number for SCFD1 rs10139154 in healthy controls and in ALS cases. HC, healthy controls; ALS, amyotrophic lateral sclerosis; SCFD1, Sec1 family domain containing 1 .

org/index.html). In a 2016 GWAS on patients with ALS, the SCFD1 rs10139154 polymorphism was initially reported as a possibly significant genetic marker (27). More precisely, in the discovery phase, a statistically significant effect size (OR, 1.09) was reported; whereas the effect size remained at the

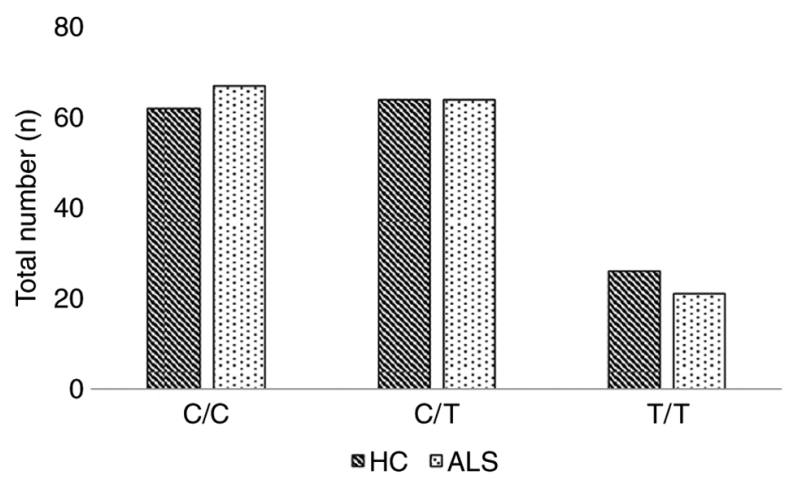

Figure 2. Genotype number for SCFD1 rs10139154 in healthy controls and in ALS cases. HC, healthy controls; ALS, amyotrophic lateral sclerosis; SCFD1, Sec1 family domain containing 1 .

replication phase $(\mathrm{OR}, 1.06)$ statistical significance was not achieved.

Additionally, Chen et al (28) reported that the SCFD1 rs10139154 polymorphism was associated with the AAO of patients with ALS of Chinese ancestry. More precisely, 


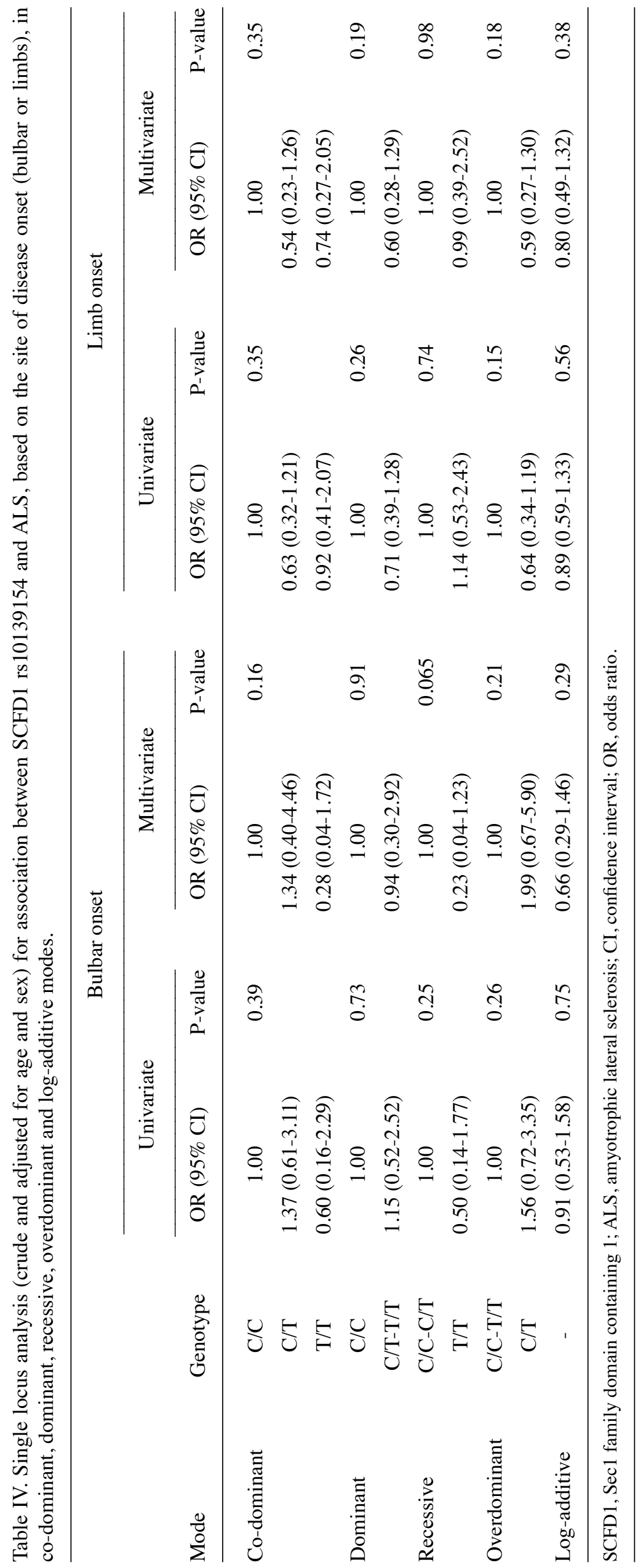


Table V. Single locus analysis (crude and adjusted for sex) for association between SCFD1 rs10139154 and AAO of ALS, in co-dominant, dominant, recessive, overdominant and log-additive mode.

\begin{tabular}{|c|c|c|c|c|c|}
\hline Mode & Genotype & Mean (SE) & Difference $(95 \% \mathrm{CI})$ & $\begin{array}{l}\text { Univariate } \\
\text { P-value }\end{array}$ & $\begin{array}{c}\text { Multivariate } \\
\text { P-value }\end{array}$ \\
\hline \multirow[t]{3}{*}{ Co-dominant } & $\mathrm{C} / \mathrm{C}$ & $63.22(1.5)$ & 0.00 & 0.7 & 0.68 \\
\hline & $\mathrm{C} / \mathrm{T}$ & $64.75(1.32)$ & $1.53(-2.38$ to 5.43$)$ & & \\
\hline & $\mathrm{T} / \mathrm{T}$ & $63.05(2.44)$ & $-0.18(-5.77$ to 5.42$)$ & & \\
\hline \multirow[t]{2}{*}{ Dominant } & $\mathrm{C} / \mathrm{C}$ & $63.22(1.5)$ & 0.00 & 0.55 & 0.58 \\
\hline & $\mathrm{C} / \mathrm{T}-\mathrm{T} / \mathrm{T}$ & $64.33(1.16)$ & $1.11(-2.54$ to 4.75$)$ & & \\
\hline \multirow[t]{2}{*}{ Recessive } & $\mathrm{C} / \mathrm{C}-\mathrm{C} / \mathrm{T}$ & $63.97(1.00)$ & 0.00 & 0.73 & 0.67 \\
\hline & $\mathrm{T} / \mathrm{T}$ & $63.05(2.44)$ & $-0.92(-6.17$ to 4.33$)$ & & \\
\hline \multirow[t]{2}{*}{ Overdominant } & $\mathrm{C} / \mathrm{C}-\mathrm{T} / \mathrm{T}$ & $63.18(1.27)$ & 0.00 & 0.4 & 0.39 \\
\hline & $\mathrm{C} / \mathrm{T}$ & $64.75(1.32)$ & $1.57(-2.09$ to 5.23$)$ & & \\
\hline Log-additive & --- & --- & $0.33(-2.26$ to 2.93$)$ & 0.8 & 0.85 \\
\hline
\end{tabular}

SCFD1, Sec1 family domain containing 1; ALS, amyotrophic lateral sclerosis; AAA, age at onset; CI, confidence interval; SE, standard error.

Table VI. Single locus analysis (crude and adjusted for sex) for association between SCFD1 rs10139154 and the AAO of ALS with bulbar onset, in co-dominant, dominant, recessive, overdominant and log-additive mode.

\begin{tabular}{|c|c|c|c|c|c|}
\hline Mode & Genotype & Mean (SE) & Difference $(95 \% \mathrm{CI})$ & $\begin{array}{l}\text { Univariate } \\
\text { P-value }\end{array}$ & $\begin{array}{c}\text { Multivariate } \\
\text { P-value }\end{array}$ \\
\hline \multirow[t]{3}{*}{ Co-dominant } & $\mathrm{C} / \mathrm{C}$ & $67.83(2.28)$ & 0.00 & \multirow[t]{3}{*}{0.46} & \multirow[t]{3}{*}{0.51} \\
\hline & $\mathrm{C} / \mathrm{T}$ & $63.12(2.81)$ & $-4.72(-12.18$ to 2.75$)$ & & \\
\hline & $\mathrm{T} / \mathrm{T}$ & $66.33(4.67)$ & $-1.50(-14.29$ to 11.29$)$ & & \\
\hline \multirow[t]{2}{*}{ Dominant } & $\mathrm{C} / \mathrm{C}$ & $67.83(2.28)$ & 0.00 & \multirow[t]{2}{*}{0.25} & \multirow[t]{2}{*}{0.28} \\
\hline & $\mathrm{C} / \mathrm{T}-\mathrm{T} / \mathrm{T}$ & $63.6(2.46)$ & $-4.23(-11.38$ to 2.91$)$ & & \\
\hline \multirow[t]{2}{*}{ Recessive } & $\mathrm{C} / \mathrm{C}-\mathrm{C} / \mathrm{T}$ & $65.07(1.92)$ & 0.00 & \multirow[t]{2}{*}{0.84} & \multirow[t]{2}{*}{0.89} \\
\hline & $\mathrm{T} / \mathrm{T}$ & $66.33(4.67)$ & $1.26(-10.86$ to 13.38$)$ & & \\
\hline \multirow[t]{2}{*}{ Overdominant } & $\mathrm{C} / \mathrm{C}-\mathrm{T} / \mathrm{T}$ & $67.53(1.98)$ & 0.00 & \multirow[t]{2}{*}{0.22} & \multirow[t]{2}{*}{0.25} \\
\hline & $\mathrm{C} / \mathrm{T}$ & $63.12(2.81)$ & $-4.42(-11.32$ to 2.49$)$ & & \\
\hline Log-additive & --- & --- & $-2.27(-7.88$ to 3.33$)$ & 0.43 & 0.44 \\
\hline
\end{tabular}

SCFD1, Sec1 family domain containing 1; ALS, amyotrophic lateral sclerosis; AAA, age at onset; CI, confidence interval; SE, standard error.

they found that carriers of the $\mathrm{C} / \mathrm{C}$ genotype had a lower AAO of ALS $(49.53 \pm 10.75$ years) compared with the $\mathrm{C} / \mathrm{T}$ carriers $(53.75 \pm 11.84$ years; $\mathrm{P}=0.002)$. Furthermore, in the recessive model analysis, $\mathrm{C} / \mathrm{C}$ carriers had a lower ALS AAO (49.53 \pm 10.75 years) compared with $(\mathrm{C} / \mathrm{T}+\mathrm{T} / \mathrm{T}$; $53.37 \pm 11.60$ years, $\mathrm{P}=0.001$ ) (28). Apart from ALS, the SCFD1 rs10139154 ' $\mathrm{T}$ ' allele has been shown to be associated with a decreased risk of Alzheimer's disease (OR, 0.63; $\mathrm{P}=0.036)$, in the recessive mode (24). However, the aforementioned results could not be replicated in the present study.

Of note, the minor allele frequency (MAF) at the study of Chen et al (28) was the ' $\mathrm{C}$ ' allele, while this was found to be the ' $\mathrm{T}$ ' allele in the present study, suggesting that ancestry may be important for the discrepancy between the studies. Based on 1,000 Genomes Project Phase 3 allele frequencies, there is a great variability at the MAF allele frequency between populations (https://www.ensembl.org/Homo_ sapiens/Variation/Population?db=core;r=14:30677792-306787
$92 ; \mathrm{v}=\mathrm{rs} 10139154 ; \mathrm{vdb}=$ variation; $\mathrm{vf}=181642009)$. In fact, while the ' $\mathrm{C}$ ' allele appears to be the MAF at East Asian and African population, the ' $\mathrm{T}$ ' allele is considered the MAF at Europeans, South Asians and Americans.

Apart from genetic variability between ethnicities, there are other possible explanations for the fact that no association was found between SCFD1 rs10139154 and ALS. For instance, the effect of a polymorphism at expression quantitative trait loci (eQTL); in fact there is evidence to suggest that variants identified in GWASs may possibly alter the risk of disease through gene regulation via eQTL (37). Concerning the rs10139154 polymorphism, it has emerged as the most significant SCFD1 polymorphism associated with ALS in a GWAS (27). In addition, for each SCFD1 rs10139154 ' $\mathrm{T}$ ' allele, an increased SCFD1 expression has been found in different tissue types obtained from genotype-tissue expression and in post-mortem control data, as demonstrated in the study by Iacoangeli et al (38). However, 
Table VII. Single locus analysis (crude and adjusted for sex) for association between SCFD1 rs10139154 and the AAO of ALS with limb onset, in co-dominant, dominant, recessive, overdominant and log-additive mode.

\begin{tabular}{|c|c|c|c|c|c|}
\hline Mode & Genotype & Mean (SE) & Difference $(95 \% \mathrm{CI})$ & $\begin{array}{l}\text { Univariate } \\
\text { P-value }\end{array}$ & $\begin{array}{c}\text { Multivariate } \\
\text { P-value }\end{array}$ \\
\hline \multirow[t]{3}{*}{ Co-dominant } & $\mathrm{C} / \mathrm{C}$ & $61.16(2.5)$ & 0.00 & 0.92 & 0.93 \\
\hline & $\mathrm{C} / \mathrm{T}$ & $62.6(2.39)$ & $1.44(-5.72$ to 8.60$)$ & & \\
\hline & $\mathrm{T} / \mathrm{T}$ & $61.17(3.61)$ & $0.01(-8.48$ to 8.49$)$ & & \\
\hline \multirow[t]{2}{*}{ Dominant } & $\mathrm{C} / \mathrm{C}$ & $61.16(2.5)$ & 0.00 & 0.78 & 0.8 \\
\hline & $\mathrm{C} / \mathrm{T}-\mathrm{T} / \mathrm{T}$ & $62.06(1.98)$ & $0.90(-5.34$ to 7.14$)$ & & \\
\hline \multirow[t]{2}{*}{ Recessive } & $\mathrm{C} / \mathrm{C}-\mathrm{C} / \mathrm{T}$ & $61.73(1.77)$ & 0.00 & 0.89 & 0.89 \\
\hline & $\mathrm{T} / \mathrm{T}$ & $61.17(3.61)$ & $-0.56(-8.51$ to 7.39$)$ & & \\
\hline \multirow[t]{2}{*}{ Overdominant } & $\mathrm{C} / \mathrm{C}-\mathrm{T} / \mathrm{T}$ & $61.16(2.05)$ & 0.00 & 0.68 & 0.7 \\
\hline & $\mathrm{C} / \mathrm{T}$ & $62.6(2.39)$ & $1.44(-5.26$ to 8.14$)$ & & \\
\hline Log-additive & - & - & $0.24(-3.82$ to 4.29$)$ & 0.91 & 0.92 \\
\hline
\end{tabular}

SCFD1, Sec1 family domain containing 1; ALS, amyotrophic lateral sclerosis; AAA, age at onset; CI, confidence interval; SE, standard error.

no alteration in SCFD1 expression with the addition of the SCFD1 rs10139154 ' $\mathrm{T}$ ' alleles was found at the post-mortem ALS cohort of that study (38). These results suggested that between the post-mortem ALS cohort and controls, different correlations existed between the SCFD1 genotype and SCFD1 eQTLs expression, which may influence the risk of the disease (38).

Currently, the available treatments for ALS are very limited, while patients with ALS usually succumb to the disease within 3-4 years from the time of diagnosis (39). Therefore, ongoing trials target precision medicine approaches, and genetic targets that will possibly influence the natural course of ALS (40). In this sense, research regarding ALS (with genetic studies included) may reveal the molecular mechanisms behind neurodegeneration, and may lead to the development of novel therapeutic agents (41).

Certain limitations of the present study need to be mentioned. Firstly, the participants with ALS were not screened for the commonest fALS genes, namely the C9orf72, SOD1, TDP-43 and FUS/TLS genes $(10,18)$. The authors also acknowledge that another constraint is that in the current statistical models, several potential cofounders (genetic and non-genetic) were not included $(15,16)$. Finally, the present study had a case-control design and thus included all the inherent limitations of such a type of study (42). On the contrary, it should be noted that a major strength of the present case-control study is the homogeneity of the entire cohort, as the data of both patients with ALS and the control group were collected from a specific geographical area of central Greece.

In conclusion, the present study found that the SCFD1 rs10139154 polymorphism was not associated with ALS. However, whether this genetic locus is among the risk factors of ALS cannot be established with absolute certitude at the moment. Bearing in mind the latter considerations, further large-scale cooperative studies examining the carriage of the SCFD1 rs10139154 polymorphism in multiethnic cohorts are of great necessity, in order for the attribute risk of this variant to ALS to be fully elucidated.

\section{Acknowledgements}

Not applicable.

\section{Funding}

The present study was supported in part by a research grant from the Research Committee of the University of Thessaly, Greece (code: 5287).

\section{Availability of data and materials}

The datasets used and/or analyzed during the current study are available from the corresponding author on reasonable request.

\section{Authors' contributions}

VS and ED were involved in the conceptualization of the study. VS, AMA, IL, CB, GN, KP and MS were involved in the study methodology and validation, in data curation and software. VS was involved in the formal analysis. VS, AMA, DPB, DAS, AT, $\mathrm{PDM}$ and ED were involved in the investigative aspects and design of the study. VS was involved in the writing and preparation of the original draft. VS, AMA, IL, CB, GN, KP, MS, DPB, DAS, AT, PDM and ED were involved in the writing, reviewing and editing of the manuscript. ED supervised the study and was involved in project administration and funding acquisition. All authors have read and approved the final manuscript. VS and ED confirm the authenticity of all the raw data.

\section{Ethics approval and consent to participate}

The research study protocol of the present study was approved by the Local Ethics Committee (University Hospital of Larissa: 59295/23-01-2017) and it was deemed in accordance to the Declaration of Helsinki. The experimental protocol was explained and all the participants provided their written informed consent in order to be included in the study, with the freedom of withdrawing from the study at any given time. 


\section{Patient consent for publication}

Not applicable.

\section{Competing interests}

DAS is the Editor-in-Chief for the journal, but had no personal involvement in the reviewing process, or any influence in terms of adjudicating on the final decision, for this article. The other authors declare that they have no competing interests.

\section{References}

1. Kojima Y,Kasai T, Noto YI, Ohmichi T, Tatebe H, Kitaoji T, Tsuji Y, Kitani-Morii F, Shinomoto M, Allsop D, et al: Amyotrophic lateral sclerosis: Correlations between fluid biomarkers of NfL, TDP-43, and tau, and clinical characteristics. PLoS One 16: e0260323, 2021.

2. Manjaly ZR, Scott KM, Abhinav K, Wijesekera L, Ganesalingam J, Goldstein LH, Janssen A, Dougherty A, Willey E, Stanton BR, et al: The sex ratio in amyotrophic lateral sclerosis: A population based study. Amyotroph Lateral Scler 11: 439-442, 2010.

3. Marin B, Boumédiene F, Logroscino G, Couratier P, Babron MC, Leutenegger AL, Copetti M, Preux PM and Beghi E: Variation in worldwide incidence of amyotrophic lateral sclerosis: A meta-analysis. Int J Epidemiol 46: 57-74, 2017.

4. Mitsumoto H, Przedborski S and Gordon PH (eds): Amyotrophic Lateral Sclerosis. CRC Press, p872, 2005.

5. Masrori P and Van Damme P: Amyotrophic lateral sclerosis: A clinical review. Eur J Neurol 27: 1918-1929, 2020.

6. Niedermeyer S, Murn M and Choi PJ: Respiratory failure in amyotrophic lateral sclerosis. Chest 155: 401-408, 2019.

7. Abramzon YA, Fratta P, Traynor BJ and Chia R: The overlapping genetics of amyotrophic lateral sclerosis and frontotemporal dementia. Front Neurosci 14: 42, 2020.

8. Ahmed RM, Devenney EM, Strikwerda-Brown C, Hodges JR, Piguet $\mathrm{O}$ and Kiernan MC: Phenotypic variability in ALS-FTD and effect on survival. Neurology 94: e2005-e2013, 2020.

9. Saberi S, Stauffer JE, Schulte DJ and Ravits J: Neuropathology of amyotrophic lateral sclerosis and its variants. Neurol Clin 33 855-876, 2015.

10. Mejzini R, Flynn LL, Pitout IL, Fletcher S, Wilton SD and Akkari PA: ALS genetics, mechanisms, and therapeutics: Where are we now? Front Neurosci 13: 1310, 2019.

11. Dardiotis E, Aloizou AM, Siokas V, Patrinos GP, Deretzi G Mitsias P, Aschner M and Tsatsakis A: The role of MicroRNAs in patients with amyotrophic lateral sclerosis. J Mol Neurosci 66: 617-628, 2018.

12. Dardiotis E, Siokas V, Sokratous M, Tsouris Z, Aloizou AM, Florou D, Dastamani M, Mentis AA and Brotis AG: Body mass index and survival from amyotrophic lateral sclerosis: A meta-analysis. Neurol Clin Pract 8: 437-444, 2018.

13. Hardiman O, Al-Chalabi A, Chio A, Corr EM, Logroscino G, Robberecht W, Shaw PJ, Simmons Z and van den Berg LH: Amyotrophic lateral sclerosis. Nat Rev Dis Primers 3: 17071, 2017.

14. Siokas V, Aloizou AM and Pateraki G: Chapter 21 - Toxicology of neurodegenerative diseases. In: Toxicological Risk Assessment and Multi-System Health Impacts from Exposure. Tsatsakis AM (ed). Academic Press, pp247-258, 2021.

15. Dardiotis E, Siokas V, Sokratous M, Tsouris Z, Michalopoulou A. Andravizou A, Dastamani M, Ralli S, Vinceti M, Tsatsakis A and Hadjigeorgiou GM: Genetic polymorphisms in amyotrophic lateral sclerosis: Evidence for implication in detoxification pathways of environmental toxicants. Environ Int 116: 122-135, 2018.

16. Tsatsakis AM (ed): Toxicological Risk Assessment and MultiSystem Health Impacts from Exposure. Elsevier, 2021.

17. Mathis S, Goizet C, Soulages A, Vallat JM and Masson GL: Genetics of amyotrophic lateral sclerosis: A review. J Neurol Sci 399: 217-226, 2019.

18. Oskarsson B, Gendron TF and Staff NP: Amyotrophic lateral sclerosis: An update for 2018. Mayo Clin Proc 93: 1617-1628, 2018.

19. Li HF and Wu ZY: Genotype-phenotype correlations of amyotrophic lateral sclerosis. Transl Neurodegener 5: 3, 2016.

20. Brown RH and Al-Chalabi A: Amyotrophic lateral sclerosis. $\mathrm{N}$ Engl J Med 377: 162-172, 2017.

21. Ghasemi M and Brown RH Jr: Genetics of amyotrophic lateral sclerosis. Cold Spring Harb Perspect Med 8: a024125, 2018
22. van Es MA, Hardiman O, Chio A, Al-Chalabi A, Pasterkamp RJ, Veldink JH and van den Berg LH: Amyotrophic lateral sclerosis. Lancet 390: 2084-2098, 2017.

23. Hou N, Yang Y, Scott IC and Lou X: The Sec domain protein Scfd1 facilitates trafficking of ECM components during chondrogenesis. Dev Biol 421: 8-15, 2017.

24. Stamati P, Siokas V, Aloizou AM, Karampinis E, Arseniou S, Rakitskii VN, Tsatsakis A, Spandidos DA, Gozes I, Mitsias PD, et al: Does SCFD1 rs10139154 polymorphism decrease Alzheimer's disease risk? J Mol Neurosci 69: 343-350, 2019.

25. Bando Y, Katayama T, Taniguchi M, Ishibashi T, Matsuo N, Ogawa S and Tohyama M: RA410/Sly1 suppresses MPP+ and 6-hydroxydopamine-induced cell death in SH-SY5Y cells. Neurobiol Dis 18: 143-151, 2005.

26. Carr CM and Rizo J: At the junction of SNARE and SM protein function. Curr Opin Cell Biol 22: 488-495, 2010.

27. van Rheenen W, Shatunov A, Dekker AM, McLaughlin RL, Diekstra FP, Pulit SL, van der Spek RA, Võsa U, de Jong S, Robinson MR, et al: Genome-wide association analyses identify new risk variants and the genetic architecture of amyotrophic lateral sclerosis. Nat Genet 48: 1043-1048, 2016.

28. Chen Y, Zhou Q, Gu X, Wei Q, Cao B, Liu H, Hou Y and Shang H: An association study between SCFD1 rs10139154 variant and amyotrophic lateral sclerosis in a Chinese cohort. Amyotroph Lateral Scler Frontotemporal Degener 19: 413-418, 2018.

29. Ludolph A, Drory V,Hardiman O, Nakano I, Ravits J, Robberecht W and Shefner J; WFN Research Group On ALS/MND: A revision of the El Escorial criteria-2015. Amyotroph Lateral Scler Frontotemporal Degener 16: 291-292, 2015.

30. Dardiotis E, Karampinis E, Siokas V, Aloizou AM, Rikos D, Ralli S, Papadimitriou D, Bogdanos DP and Hadjigeorgiou GM: ERCC6L2 rs591486 polymorphism and risk for amyotrophic lateral sclerosis in Greek population. Neurol Sci 40: 1237-1244, 2019.

31. Siokas V, Aloizou AM, Liampas I, Tsouris Z, Mentis AA, Nasios G, Papadimitriou D, Bogdanos DP, Hadjigeorgiou GM and Dardiotis E: Lack of association between TREM2 rs75932628 variant and amyotrophic lateral sclerosis. Mol Biol Rep 48: 2601-2610, 2021.

32. Siokas V, Karampinis E, Aloizou AM, Mentis AA, Liakos P Papadimitriou D, Liampas I, Nasios G, Bogdanos DP, Hadjigeorgiou GM and Dardiotis E: CYP1A2 rs762551 polymorphism and risk for amyotrophic lateral sclerosis. Neurol Sci 42: 175-182, 2021.

33. Liampas I, Siokas V, Aloizou AM, Bakirtzis C, Tsouris Z, Nousia A Nasios G, Papadimitriou D, Liakos P, Bogdanos DP, et al: MOBP rs616147 polymorphism and risk of amyotrophic lateral sclerosis in a greek population: A case-control study. Medicina (Kaunas) 57: $1337,2021$.

34. Siokas V, Kardaras D, Aloizou AM, Asproudis I, Boboridis KG, Papageorgiou E, Hadjigeorgiou GM, Tsironi EE and Dardiotis E: BDNF rs6265 (Val66Met) polymorphism as a risk factor for blepharospasm. Neuromolecular Med 21: 68-74, 2019.

35. Siokas V, Aslanidou P, Aloizou AM, Peristeri E, Stamati P, Liampas I, Arseniou S, Drakoulis N, Aschner M, Tsatsakis A, et al: Does the CD33 rs3865444 polymorphism confer susceptibility to Alzheimer's disease? J Mol Neurosci 70: 851-860, 2020.

36. Solé X, Guinó E, Valls J, Iniesta R and Moreno V: SNPStats: A web tool for the analysis of association studies. Bioinformatics 22: 1928-1929, 2006.

37. GTEx Consortium: The genotype-tissue expression (GTEx) project. Nat Genet 45: 580-585, 2013.

38. Iacoangeli A, Fogh I, Selvackadunco S, Topp SD, Shatunov A, van Rheenen W, Al-Khleifat A, Opie-Martin S, Ratti A, Calvo A, et al: SCFD1 expression quantitative trait loci in amyotrophic lateral sclerosis are differentially expressed. Brain Commun 3: fcab236, 2021.

39. Keon M, Musrie B, Dinger M, Brennan SE, Santos J and Saksena NK: Destination amyotrophic lateral sclerosis. Front Neurol 12: 596006, 2021

40. Kiernan MC, Vucic S, Talbot K, McDermott CJ, Hardiman O, Shefner JM, Al-Chalabi A, Huynh W, Cudkowicz M, Talman P, et al: Improving clinical trial outcomes in amyotrophic lateral sclerosis. Nat Rev Neurol 17: 104-118, 2021.

41. Amado DA and Davidson BL: Gene therapy for ALS: A review. Mol Ther 29: 3345-3358, 2021.

42. Yuan W, Beaulieu-Jones BK, Yu KH, Lipnick SL, Palmer N, Loscalzo J, Cai T and Kohane IS: Temporal bias in case-control design: Preventing reliable predictions of the future. NatCommun 12: 1107, 2021.

(c) (i) $-(9)$ This work is licensed under a Creative Commons International (CC BY-NC-ND 4.0) License. 